\title{
Coherent phonon dynamics at the martensitic phase transition of $\mathrm{Ni}_{2} \mathrm{MnGa}$
}

\author{
S. O. Mariager, ${ }^{\text {a) }}$ A. Caviezel, P. Beaud, C. Quitmann, and G. Ingold \\ Swiss Light Source, Paul Scherrer Institut, 5232 Villigen, Switzerland
}

(Received 29 April 2012; accepted 29 May 2012; published online 29 June 2012)

\begin{abstract}
We use time-resolved optical reflectivity to study the laser stimulated dynamics in the magnetic shape memory alloy $\mathrm{Ni}_{2} \mathrm{MnGa}$. We observe two coherent optical phonons, at $1.2 \mathrm{THz}$ in the martensite phase and at $0.7 \mathrm{THz}$ in the pre-martensite phase, which we interpret as a zone-folded acoustic phonon and a heavily damped amplitudon, respectively. In the martensite phase the martensitic phase transition can be induced by a fs laser pulse on a timescale of a few ps. (C) 2012 American Institute of Physics. [http://dx.doi.org/10.1063/1.4730946]
\end{abstract}

In magnetic shape memory Heusler alloys the combination of ferromagnetism and a structural martensitic phase transition leads to large magnetic field induced strains, reaching up to $10 \%$ in the prototype $\mathrm{Ni}_{2} \mathrm{MnGa}$. ${ }^{1}$ While practical applications already exist, both the structure of the martensite phase $(\mathrm{MT})^{3}$ and the microscopic origin of the phase transition remain under discussion. ${ }^{2,4}$ Upon cooling $\mathrm{Ni}_{2} \mathrm{MnGa}$ undergoes two structural phase transitions. At $T_{\mathrm{PMT}} \approx 260 \mathrm{~K}$ a pre-martensitic transition leads to a gradual modulation of the high temperature cubic austenite phase (AUS) of L $2{ }_{1}$ symmetry to the pre-martensite phase (PMT). The subsequent first order martensitic transition occurs at $T_{\mathrm{MT}} \approx 220 \mathrm{~K}$. Through both structural transitions $\mathrm{Ni}_{2} \mathrm{MnGa}$ remains ferromagnetic $\left(T_{C} \approx 380 \mathrm{~K}\right)$.

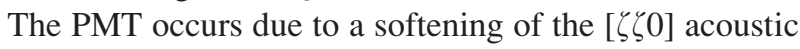
phonon mode at $\zeta_{0}=1 / 3$. The resulting structure arises from a periodic modulation of the positions of the atoms within the cubic unit cell tripling the unit cell along $\{110\} .^{4-6}$ The structure of the MT is on the other hand debated, in part because different stoichiometries lead to different structures. ${ }^{7}$ The reported structures can roughly be summarized as tetragonal or orthorhombic distortions of the AUS unit cell, combined with a 5 or 7 fold distortion along $\{110\}$. The latter distortion has been described both as a periodic modulation and a shuffling of atomic layers. ${ }^{8}$ A possible explanation was given recently when the MT structure of $\mathrm{Ni}_{2} \mathrm{MnGa}$ was described as an adaptive phase, ${ }^{3,9}$ where nanotwinning occurs along $\{110\}$ in order to minimize the elastic energy at the cubic/tetragonal interface. As a result there are 12 tetragonal twins ${ }^{10}$ and the 5 and 7 fold distortions are $(3 \overline{2})_{2}$ and $(5 \overline{2})_{2}$ in Zhadonov notation. ${ }^{11}$ Also the driving forces of the martensitic transition are not fully understood on the microscopic level. The electronic stabilization appears to be a pseudogap $0.3 \mathrm{eV}$ below the Fermi level. The pseudogap has been measured with photo-emission ${ }^{2}$ and magneto-resistance $^{12}$ and calculated theoretically. ${ }^{13,14}$ It forms by redistribution of minority spin Ni-3d states and can be understood as a band Jahn-Teller effect. In addition it has recently been stressed that the combined magnetic and vibrational excitations must be included in order to reproduce the full phase diagram of $\mathrm{Ni}_{2} \mathrm{MnGa}^{4}$, while coupling between acoustic and

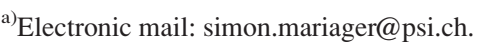

optical phonon modes in the AUS can account for the wavevectors related to the modulation. ${ }^{15}$

Motivated by the fact that fs laser pulses can excite coherent optical phonons ${ }^{16}$ and induce structural phase transitions through the release of Jahn-Teller distortions ${ }^{17}$ we studied the phonon modes and structural dynamics in $\mathrm{Ni}_{2} \mathrm{MnGa}$ by all optical time-resolved reflectivity. In this letter we show that low fluence excitations of the different phases excite two distinct coherent optical phonons in the PMT and MT, respectively. Second, the martensitic phase transition can be induced, leading to signatures of the PMT appearing after a few ps.

The samples were a stoichiometric $\mathrm{Ni}_{2} \mathrm{MnGa}$ crystal $\left(10 \times 10 \times 1 \mathrm{~mm}^{3}\right)$ and non-stochiometric $\mathrm{Ni}_{50} \mathrm{Mn}_{28.3} \mathrm{Ga}_{21.7}$ crystal $\left(4 \times 4 \times 1 \mathrm{~mm}^{3}\right)$ (both from AdaptaMat Ltd.). Both crystals were cut and polished with $\{001\}$ facets. The photoinduced change in reflectivity was investigated in an optical pump-probe setup with a time resolution of $\sim 80 \mathrm{fs}$. The $50 \mathrm{fs}$ $800 \mathrm{~nm}$ pump and probe pulse were generated with a $2 \mathrm{kHz}$ Ti-Saphire laser system. The pump and probe pulses were cross polarized and incident along the [001] surface normal with the probe polarization aligned to the [100] crystal axis. The pump was focused to $500 \times 500 \mu \mathrm{m}^{2}$, and the probe was a factor of three smaller to ensure homogenous excitation of the probe region. The sample was mounted in a cryostat.

To verify the existence of the MT the magnetic AC susceptibility was measured as a function of sample temperature. The measurements were performed with a Quantum Design PPMS with a driving field of 10 Oe and no applied static field. The results are independent of frequency between

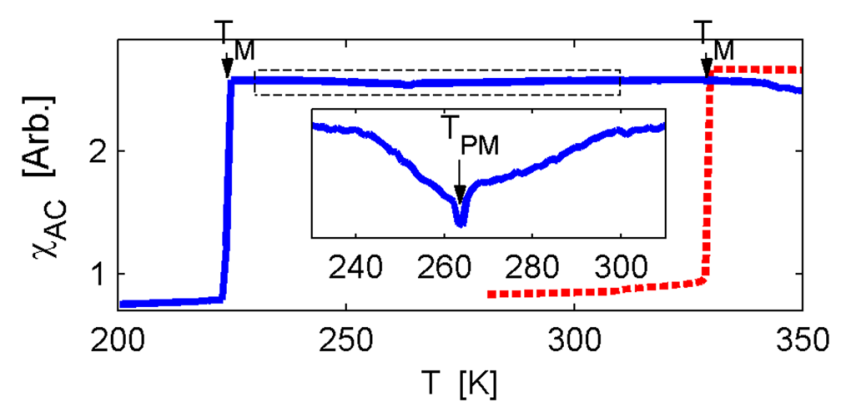

FIG. 1. AC magnetization curves for the stoichiometric (blue, solid) and non-stoichiometric (red, dashed) $\mathrm{Ni}_{2} \mathrm{MnGa}$ crystals. The inset highlights the pre-martensite region and corresponds to the dashed box in the main figure. 
13 and $3500 \mathrm{~Hz}$ and shown in Fig. 1 for $34 \mathrm{~Hz}$. The Martensitic transition is visible as a sharp change in susceptibility at $T_{\mathrm{MT}}=224 \mathrm{~K}$ and $T_{\mathrm{MT}}=329 \mathrm{~K}$ for the two crystals, respectively. The change in susceptibility is due to the lower crystal symmetry and higher magnetocrystalline anisotropy of the MT. For the stoichiometric crystal the non-magnetic premartensitic transition is visible in the inset at $T_{\mathrm{PMT}}=264 \mathrm{~K}$. The magnetic susceptibilities in Fig. 1 are consistent with the literature. ${ }^{1,2}$

In Fig. 2 we show the relative change in reflectivity as a function of time after laser excitation for the stoichiometric crystal at temperatures between 170 and $280 \mathrm{~K}$. In the MT $(T<215 \mathrm{~K})$ a strong oscillation with a low damping is seen in the transient reflectivity. This is the signature of a coherent optical phonon. ${ }^{16}$ Above $215 \mathrm{~K}$ the signal is markedly different. At intermediate temperatures $(220-260 \mathrm{~K})$ an oscillation is still visible, but the frequency is lower and the stronger damping quenches the oscillation after a single period. As the temperature is increased the damping gets stronger and at $270 \mathrm{~K}$ the oscillation is gone. Here the change in reflectivity is reduced to the incoherent response of a metal, due to the heating and thermalization of the electron gas and the lattice. ${ }^{18}$ The absence of coherent phonons in the AUS is not surprising. In opaque materials fs laser pulses mainly excite symmetry conserving $\mathrm{A}_{1 g}$ modes by displacive excitation, and no such modes exist in the AUS of $\mathrm{Ni}_{2} \mathrm{MnGa} .{ }^{15}$ From Fig. 2 it is obvious that transient reflectivity probes the phase diagram of $\mathrm{Ni}_{2} \mathrm{MnGa}$. The transition temperatures $T_{\mathrm{MT}}$ and $T_{\mathrm{PMT}}$ agree with those in Fig. 1 and the slight shift to lower temperatures is due to the hysteresis of the first order phase transition. The susceptibility was measured during heating and the reflectivity during cooling. We find the same pho-

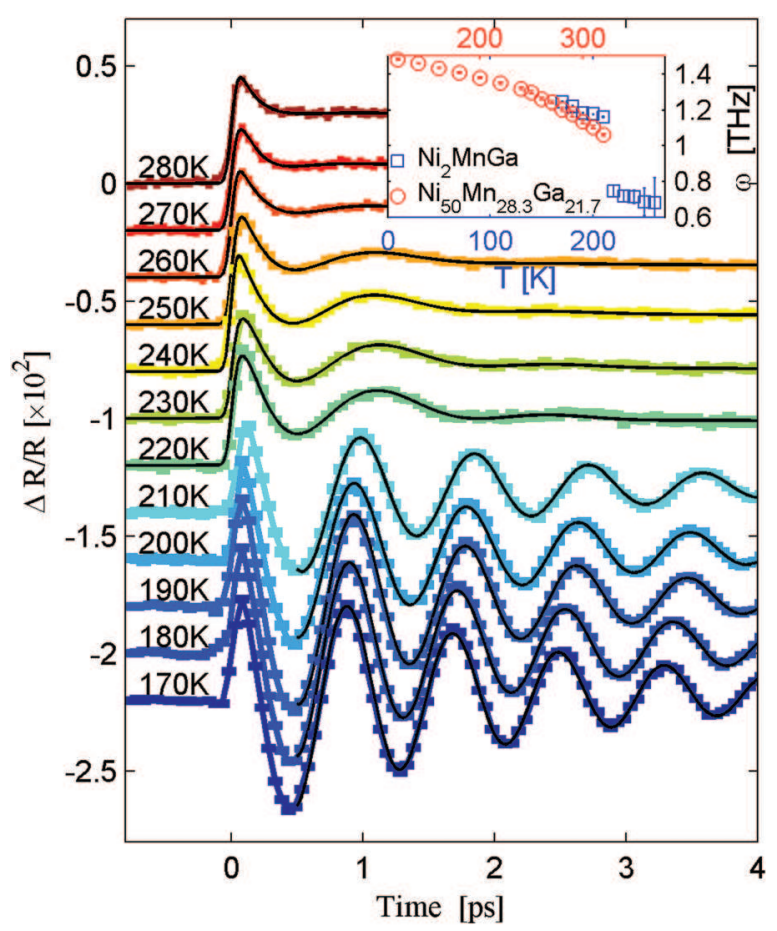

FIG. 2. Time resolved reflectivity on $\mathrm{Ni}_{2} \mathrm{MnGa}$ obtained with a laser pump power of $1 \mathrm{~mJ} / \mathrm{cm}^{2}$. Black lines are fits to the data. The insert shows the frequencies in both phases as a function of temperature (blue squares), and also includes data from the non-stoichiometric crystal (red circles). nons in the non-stoichiometric crystal, though the damping in the PMT is stronger. This is due either to the higher temperature or the stoichiometry.

To extract the phonon frequencies we fit the data from the MT with a single damped cosine function $\Delta R_{\text {osc }}=A \cos (2 \pi \omega t+\Phi) e^{-t / \tau}$. To minimize the number of free parameters we ignore the initial peak, which as explained below originates from the heated electrons, by restricting the fit to $t>0.5 \mathrm{ps}$. For the PMT we fit the entire curve and account for the incoherent part by using $\Delta R=\Delta R_{\text {osc }}+\Delta R_{e}$ with

$$
\Delta R_{e}(t)=a\left(1-e^{-t / \tau_{e e}}\right) e^{-t / \tau_{e p}}+b\left(1-e^{-t / \tau_{e p}}\right) e^{-t / \tau_{t h}},
$$

where $\tau_{e e}$ and $\tau_{e p}(\approx 0.8 \mathrm{ps})$ are the electron-electron and electron-phonon relaxation times and $\tau_{t h}$ describes the subsequent cooling of the lattice. The constants $a$ and $b$ determine the ratio between the electronic peak and the thermal contribution. The fit function was convoluted with a Gaussian accounting for the $80 \mathrm{fs}$ time-resolution, and the resulting fits are shown in Fig. 2. In the inset the frequencies $\omega$ are plotted as a function of temperature with error bars given by $95 \%$ confidence intervals from the fits. Because of the high damping in the PMT of the non-stoichiometric crystal, this frequency could not be extracted at a fluence of $1 \mathrm{~mJ} / \mathrm{cm}^{2}$. In both phases the frequency softens with increasing temperature. Thus thermal expansion rather than anharmonic effects dominates the temperature dependence of the frequencies. We note that due to the long wavelength of the optical light compared to the crystals lattice constant the momentum transfer is $\sim 0$ and the phonon frequencies are measured at the $\Gamma$ point of the Brillouin zone.

The acoustic phonon dispersion curves of $\mathrm{Ni}_{2} \mathrm{MnGa}$ have been measured by inelastic neutron scattering, ${ }^{19}$ but we are not aware of any experimental results on optical phonons. The phonon dispersion curves have been calculated for AUS, PMT, and MT. ${ }^{4,5}$ For the non-modulated lattices the lowest optical phonon modes have frequencies above $4 \mathrm{THz}$ in the zone-center and cannot explain the observed frequencies. The structural modulations in the PMT and MT however multiply the unit cell along $\{110\}$, leading to a reduced Brillouin zone and zone folded phonons. Since the measured phonon frequencies in both phases are lower than the calculated frequencies of the non-modulated unit cells, the phonon modes must be related to the modulation. For the PMT low lying phonon modes consistent with our measurements have indeed been predicted by considering the complete tripled unit cell. ${ }^{4}$

The crystal structure of the PMT is a periodic modulation of the cubic unit cell along $\{110\}$ with a period of $3 \mathrm{a} / \sqrt{2}$. The specific phonon mode corresponding to the oscillation of the modulation amplitude is termed an amplitudon. A factor group analysis ${ }^{20}$ using the modulated structure of the PMT (Ref. 6) confirms the existence of a Raman active symmetry conserving $\mathrm{A}_{1 g}$ mode corresponding to the amplitudon. The phase of the phonon is difficult to determine due to the strong damping and overlap in time with the electronic peak. We find that $\phi=0.59 \pm .15 \mathrm{rad}$ at $1 \mathrm{~mJ} / \mathrm{cm}^{2}$ and goes to zero at higher pump fluences. This indicates a displacive excitation similar to the well known laser excitation of the $\mathrm{A}_{1 g}$ mode of $\mathrm{Bi}^{21}$ Based on the low frequency, the factor 
group analysis and the displacive character of the excitation, we suggest that the optical phonon mode observed in the PMT is the amplitudon of the modulated unit cell.

The phonon modes of the MT phase are also intimately related to the structure, which as discussed is not unambiguously clarified in the literature. X-ray diffraction data from the crystals used in this study is consistent with an adaptive modulation. These results which will be reported elsewhere confirm the 12-fold splitting of a [202] $]_{c}$ Bragg peak in the MT (Ref. 3) and the existence of superlattice peaks corresponding to a 7-fold structure. ${ }^{11}$ In a nanotwinned structure the observed low frequency coherent phonons are truly zonefolded acoustic phonons, similar to the coherent oscillations observed in superlattices. ${ }^{22}$ At the Brillouin zone center, the frequency $2 d / v$ of the first mode is roughly given by the speed of sound $v$ divided by the period of the nanotwin super cell $d$. The factor of 2 arises because a single nano-twinned cell has is bound on both sides. Using elastic constants calculated for the tetragonal structure of the MT phase, ${ }^{23}$ we find $v_{[110]}=6.07 \mathrm{~km} / \mathrm{s}$ for the mainly longitudinal wave, close to the $5.54 \mathrm{~km} / \mathrm{s}$ in the AUS phase. ${ }^{24}$ The period of the nanotwin super cell is $d \approx 21 \AA$ (Ref. 3) giving a frequency of 1.4 THz. Considering the uncertainty of the material constants and that we apply continuum theory in the microscopic limit, this is in good agreement with the frequencies found in Fig. 2. The phase of the oscillation is $\phi=5.6 \mathrm{rad}$, which corresponds to a cosine oscillation starting $\sim 100 \mathrm{fs}$ after time zero. This suggests a displacive excitation, and the slight delay with respect to time zero could indicate that the driving force of the phonon mode is thermal strain ${ }^{25}$ with the coherent phonon only being excited once the electrons and lattice starts to thermalize. Since the structure of $\mathrm{Ni}_{2} \mathrm{MnGa}$ in the MT is debated an alternative explanation of the observed coherent phonon is possible. If a periodic modulation of the tetragonal unit cell does exist, the observed phonon could be an amplitudon as for the PMT. The existence of a phason, which is the acoustic variant of the optical amplitudon, has previously been reported. ${ }^{26}$ With optical reflectivity we cannot distinguish with certainty between the two explanations, but the structure of the samples as determined by x-ray diffraction and the accuracy of the estimated frequency strongly support the hypothesis of a zone-folded acoustic phonon.

We finally consider the possibility of inducing the martensitic phase transition with a fs laser pulse on an ultrafast timescale. In Fig. 3(a) we show the transient reflectivity curves obtained in the MT at $T=200 \mathrm{~K}$ just below $T_{\mathrm{MT}}$ as a function of pump fluences from 0.5 to $5 \mathrm{~mJ} / \mathrm{cm}^{2}$. The amplitude of the initial peak at $t=0.08 \mathrm{ps}$ is linear in fluence as shown in Fig. 3(b), where the solid lines are fits to the data. This linear dependence is consistent with an electronic contribution from the laser heated electron gas, with a temperature proportional to the fluence. In contrast the change in reflectivity at $t=8 \mathrm{ps}$ shows a highly non-linear fluence dependence, with a clear increase in $\Delta \mathrm{R}$ above $\sim 3 \mathrm{~mJ} / \mathrm{cm}^{2}$. This threshold behavior is one indication of a laser induced phase transition, which for $\mathrm{Ni}_{2} \mathrm{MnGa}$ at $T=200 \mathrm{~K}$ must be attributed to the martensitic transition. To confirm this we consider in detail the transient reflectivity curves in Fig. 3(a). At low fluences $\left(<2 \mathrm{~mJ} / \mathrm{cm}^{2}\right)$ the amplitude and the damping of the zone folded acoustic mode both increase with fluence.
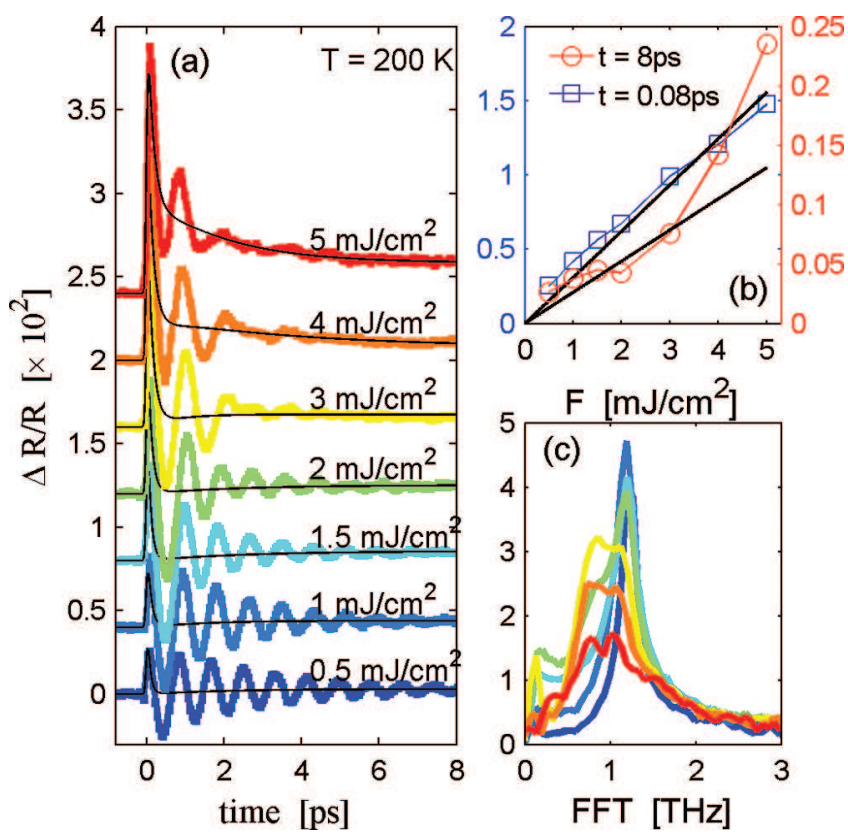

FIG. 3. (a) Transient reflectivity curves for different fluences at sample temperature $T=200 \mathrm{~K}$. The solid lines are a fit with $\Delta R_{e}$. (b) $\Delta R / R$ at $t=0.08$ and 4 ps. Black lines are linear fits. (c) Fast Fourier transform of the oscillatory part of the curves from (a), applying the same color scale.

At higher fluences a beating of the signal indicates the presence of a second frequency. To extract the frequencies we isolate the oscillatory part of the curves by subtracting the incoherent part given by $\Delta R_{e}$. The remaining oscillating signal is Fourier transformed as shown in Fig. 3(c). At low fluences the result is a single frequency at $\sim 1.2 \mathrm{THz}$ as expected. As the fluence is increased a second peak gradually arises at $\sim 0.8 \mathrm{THz}$. This frequency is consistent with the frequencies found for the amplitudon in the PMT. It shows that with increasing laser fluence the martensitic transition is induced, resulting in a change of structure from MT to PMT, with the modulation of the PMT realized through the amplitudon. The presence of a full period of the zone-folded acoustic phonon at all applied fluences is a clear proof that the nanotwinned structure remains present at early times $(t<2 \mathrm{ps})$. The beating on the other hand reveals that a significant fraction of the PMT is present after 2 ps and confirms the expected phase coexistence of PMT and MT. We have previously observed phase coexistence in a laser induced first order phase transition. ${ }^{27}$

To summarize we have measured two coherent optical phonons in the pre-martensite and martensite phases of the Heusler alloy $\mathrm{Ni}_{2} \mathrm{MnGa}$, respectively. These were characterized as an amplitudon in the PMT and a zone-folded acoustic mode arising from the nano-twinned structure in the MT. The good estimate of the frequency of the zone-folded acoustic mode supports the idea of an adaptive martensitic phase in $\mathrm{Ni}_{2} \mathrm{MnGa}$. The martensitic phase transition can be induced on a timescale of $\sim 2 \mathrm{ps}$ by laser excitation and exhibits phase coexistence between the MT and PMT phases. A confirmation of our findings will require further experiments. This can be done by measuring the optical branches of the phonon dispersion curves with inelastic neutron scattering, or by using time-resolved x-ray diffraction to directly measure the atomic motion in the time domain. 
We thank M. Medarde for help with the magnetization measurements. This work was supported by the Swiss National Foundation through NCCR MUST.

${ }^{1}$ A. Sozinov, A. A. Likhachev, N. Lanska, and K. Ullakko, Appl. Phys. Lett. 80, 1746 (2002).

${ }^{2}$ C. P. Opeil, B. Mihaila, R. K. Schulze, L. Mañosa, A. Planes, W. L. Hults, R. A. Fisher, P. S. Riseborough, P. B. Littlewood, J. L. Smith et al., Phys. Rev. Lett. 100, 165703 (2008).

${ }^{3}$ S. Kaufmann, U. K. Rößler, O. Heczko, M. Wuttig, J. Buschbeck, L. Schultz, and S. Fähler, Phys. Rev. Lett. 104, 145702 (2010).

${ }^{4}$ M. A. Uijttewaal, T. Hickel, J. Neugebauer, M. E. Gruner, and P. Entel, Phys. Rev. Lett. 102, 035702 (2009).

${ }^{5}$ A. T. Zayak, P. Entel, J. Enkovaara, A. Ayuela, and R. M. Nieminen, Phys. Rev. B 68, 132402 (2003).

${ }^{6}$ P. J. Brown, J. Crangle, T. Kanomata, M. Matsumoto, K.-U. Neumann, B. Ouladdiaf, and K. R. A. Ziebeck, J. Phys.: Condens. Matter 14, 10159 (2002).

${ }^{7}$ N. Lanska, O. Sderberg, A. Sozinov, Y. Ge, K. Ullakko, and V. K. Lindroos, J. Appl. Phys. 95, 8074 (2004).

${ }^{8}$ J. Pons, R. Santamarta, V. A. Chernenko, and E. Cesari, J. Appl. Phys. 97, 083516 (2005).

${ }^{9}$ A. G. Khachaturyan, S. M. Shapiro, and S. Semenovskaya, Phys. Rev. B 43, 10832 (1991).

${ }^{10}$ G. Jakob, T. Eichhorn, M. Kallmayer, and H. J. Elmers, Phys. Rev. B 76, 174407 (2007).

${ }^{11}$ A. Ustinov, L. Olikhovska, N. Glavatska, and I. Glavatskyy, J. Appl. Cryst. 42, 211 (2009).

${ }^{12}$ C. P. Opeil, J. C. Lashley, and J. L. Smith, J. Phys.: Conf. Ser. 150, 042109 (2009).
${ }^{13}$ A. Ayuela, J. Enkovaara, and R. M. Nieminen, J. Phys. Condens. Matter 14, 5325 (2002).

${ }^{14}$ P. Entel, M. E. Gruner, A. Dannenberg, M. Siewert, S. K. Nayak, H. C. Herper, and V. D. Buchelnikov, Mater. Sci. Forum 635, 3 (2010).

${ }^{15}$ A. T. Zayak, P. Entel, K. M. Rabe, W. A. Adeagbo, and M. Acet, Phys. Rev. B 72, 054113 (2005).

${ }^{16} \mathrm{~K}$. Ishioka and O. V. Misochko, Coherent Lattice Ocillations in Solids and Their Optical Control Part I. Fundamentals and Optical Detection Techniques (Springer, 2010), Chap. 2, pp. 23-46.

${ }^{17}$ P. Beaud, S. L. Johnson, E. Vorobeva, U. Staub, R. A. D. Souza, C. J. Milne, Q. X. Jia, and G. Ingold, Phys. Rev. Lett. 103, 155702 (2009).

${ }^{18}$ J. Hohlfeld, S.-S. Wellershoff, J. Gudde, U. Conrad, V. Jahnke, and E. Matthias, Chem. Phys. 251, 237 (2000).

${ }^{19}$ A. Zheludev, S. M. Shapiro, P. Wochner, and L. E. Tanner, Phys. Rev. B 54, 15045 (1996).

${ }^{20}$ E. Kroumova, M. I. Aroyo, J. M. Perez-Mato, A. Kirov, C. Capillas, S. Ivantchev, and H. Wondratschek, Phase Trans. 76, 155 (2003).

${ }^{21}$ K. Sokolowski-Tinten, C. Blome, J. Blums, A. Cavalleri, C. Dietrich, A. Tarasevitch, I. Uschmann, E. Frster, M. Kammler, M. H. von Hoegen et al., Nature (London) 422, 287 (2003).

${ }^{22}$ M. Bargheer, N. Zhavoronkov, Y. Gritsai, J. C. Woo, D. S. Kim, M. Woerner, and T. Elsaesser, Science 306, 1771 (2004).

${ }^{23}$ C.-M. Li, H.-B. Luo, Q.-M. Hu, R. Yang, B. Johansson, and L. Vitos, Phys. Rev. B 84, 174117 (2011).

${ }^{24}$ J. Worgull, E. Petti, and J. Trivisonno, Phys. Rev. B 54, 15695 (1996).

${ }^{25}$ C. Thomsen, H. T. Grahn, H. J. Maris, and J. Tauc, Phys. Rev. B 34, 4129 (1986).

${ }^{26}$ S. M. Shapiro, P. Vorderwisch, K. Habicht, K. Hradil, and H. Schneider, Europhys. Lett. 77, 56004 (2007).

${ }^{27}$ S. O. Mariager, F. Pressacco, G. Ingold, A. Caviezel, E. Möhr-Vorobeva, P. Beaud, S. L. Johnson, C. J. Milne, E. Mancini, S. Moyerman et al., Phys. Rev. Lett. 108, 087201 (2012). 